\title{
3D Electron diffraction for the discovery of new crystal forms of APIs
}

\author{
A. E. Lanza ${ }^{1}$, J. Potticary ${ }^{2}$, C. Hall ${ }^{2}$, V. Hamilton ${ }^{2}$, S.R. Hall ${ }^{2}$, G. Santiso-Quinones ${ }^{1}$, G. Steinfeld ${ }^{1}$, E. Hovestreydt ${ }^{1}$ \\ ${ }^{1}$ ELDICO Scientific AG, 5234 Villigen, Switzerland, \\ ${ }^{2}$ School of Chemistry, University of Bristol, Cantock's Close, Bristol, BS8 1TS, United Kingdom
}

lanza@eldico.ch

Most pharmaceuticals are administered in solid form, therefore extensive research and development efforts are invested to explore the phase diagram of APIs and solid-state formulations. The elucidation of the crystal structure of crystalline forms is the key tool for assessing their applicability, as it allows the rationalization of their relevant physicochemical properties, bioavailability, manufacturing, stability etc. Nonconventional crystallization methods[1] enable to discover new solid forms including polymorphs, cocrystals and solvates, allowing to see even known APIs under a new light. However, these methods can easily lead to nanocrystalline products, which hamper structural elucidation.

3D Electron diffraction (3D ED)[2] has recently emerged as a powerful tool for the discovery of new crystalline forms of pharmaceutical compounds,[3-5] as it allows to bypass many of the common bottlenecks of this process and of the established characterization methods based on x-ray diffraction. Small crystal size, mixture of phases, small product quantities are very frequent obstacles to the structural characterization of APIs that can be easily overcome by 3D ED methods.

Here we showcase how our electron diffractometer, fully dedicated to 3D ED experiments, represents a revolutionary innovation for the discovery of new crystal forms of APIs. Our recent results of representative case studies dealing with challenging pharmaceutical compounds will demonstrate the performances of a dedicated device and how it can meet the growing needs of the crystallographic and pharmaceutical community.

[1] Potticary, J., Hall, C., Hamilton, V., McCabe, J. F. \& Hall, S. R. (2020). Cryst. Growth Des. $20,2877$.

[2] Gemmi, M., Mugnaioli, E., Gorelik, T. E., Kolb, U., Palatinus, L., Boullay, P., Hovmöller, S. \& Abrahams, J. P. (2019). ACS Cent. Sci. 5, 1315.

[3] Andrusenko, I., Potticary, J., Hall, S. R. \& Gemmi, M. (2020). Acta Cryst. B76, 1036.

[4] Hamilton, V., Andrusenko, I., Potticary, J., Hall, C., Stenner, R., Mugnaioli, E., Lanza, A. E., Gemmi, M. \& Hall, S. R. (2020) Cryst. Growth Des. 20, 4731.

[5] Andrusenko, I., Hamilton, V., Mugnaioli E., Lanza, A., Hall, C., Potticary, J., Hall, S. R. \& Gemmi, M. (2019). Angew. Chem. Int. Ed. 58, 10919.

Keywords: Electron diffraction; pharmaceuticals; nanocrystals; co-crystals; solvates;

We gratefully acknowledge Dr. Mauro Gemmi and Dr. Iryna Andrusenko (Center for Nanotechnology Innovation @NEST, Istituto Italiano di Tecnologia, Pisa, Italy) for fruitful discussions. 